\title{
EDUCACIÓN, DESIGUALDAD Y DESPLAZAMIENTO FORZADO EN COLOMBIA*
}

\author{
LUIS EDUARDO SANDOVAL", SANDRA LILIANA BOTÓN"*** \\ \& MARÍA IRMA BOTERO \\ UNIVERSIDAD MILITAR NUEVA GRANADA
}

Recibido/ Received/ Recebido: 13/04/2010 - Aceptado/ Accepted/Aprovado: 31/08/2010

\begin{abstract}
Resumen
El documento pretende establecer la relación entre niveles socioeconómicos y educación en los hogares victimas del desplazamiento forzado e Colombia, respecto a las condiciones de conflicto armado y pobreza que se presentan a nivel departamental a partir de las características que presentan estas familias. Para esto, se realizaron estimaciones con variables de conflicto, pobreza y nivel económico de los departamentos como determinantes del grado de expulsión. Se llega a la conclusión que este fenómeno tiene mayor concentración en aquellas zonas donde la población es más vulnerable respecto a la pobreza y condiciones sociales presentes en la regiones.

Palabras clave: Desplazamiento forzado, economía de la defensa, educación, conflicto armado.
\end{abstract}

\section{EDUCATION, INEQUALITY AND FORCED DISPLACEMENT IN COLOMBIA}

\begin{abstract}
This paper seeks to establish the relationship among socioeconomic levels and education in households victimized by forced displacement in Colombia, considering armed conflict and poverty conditions at departmental level from households characteristics. In order to achieve this, some calculations were made with variables of conflict, poverty and economic level of the departments such as expulsion determinants. The conclusion is that, this phenomenon has major concentration in those zones where the population in more vulnerable in relation to poverty and regional social conditions.
\end{abstract}

Keywords: Forced displacement, defense economy, education, armed conflict.

Este artículo es resultado del proyecto de investigación ECO-568 - "Impactos socio-demollustracións del Desplazamiento Forzado en Colombia. Una evidencia para el periodo 1993-2008", financiado por la Universidad Militar "Nueva Granada".

" Docente programa de economía de la Facultad de Ciencias Económicas Universidad Militar Nueva Granada. Correo electrónico: luis.sandoval@unimilitar.edu.co.

Docente programa de economía de de la Facultad de Ciencias Económicas Universidad Militar Nueva Granada. Correo electrónico: sandra.boton@unimilitar.edu.co.

Docente programa de economía de la de la Facultad de Ciencias Económicas Universidad Militar Nueva Granada. Correo electrónico: maria.botero@unimilitar.edu.co. 


\title{
EDUCAÇÃO, DESIGUALDADE E DESLOCAMENTO FORÇADO NA COLÔMBIA
}

\begin{abstract}
Resumo
O documento visa estabelecer a relação entre os níveis socioeconômico e educacional em famílias vítimas do deslocamento forçado na Colômbia, com base nas características de pobreza mostradas por estas famílias nos departamentos que sofrem o conflito armado. Como determinantes do grau de deslocamento, estudaram-se as variáveis conflito, pobreza e nível econômico nos departamentos. A conclusão tirada é que este fenômeno é mais concentração nas regiões onde a população é mais vulnerável à pobreza $e$ às condições sociais existentes.
\end{abstract}

Palavras chave: Deslocamento forçado, economia da defesa, educação, conflito armado.

Sandoval, L., Botón, S \& Botero, M. (2011). Educación, desigualdad y desplazamiento forzado en Colombia. En: Revista de la Facultad de Ciencias Económicas de la Universidad Militar Nueva Granada. rev.fac.cienc.econ, XIX (1)

JEL: R23, D74

\section{Introducción}

La ley 387 de $1997^{1}$, ha promovido la adopción de medidas de prevención del desplazamiento forzado por parte del estado pero no ha sido suficiente para contrarrestar el número de personas desplazadas, perdida de activos, desintegración familiar, altas tasas de desempleo, deterioro de la calidad de vida, entre otros (González, 2002; Mooney, 2005; Springer, 2006; Ibáñez, 2006).

El desplazamiento, abarca situaciones de desigualdad, vulnerabilidad y ausencia del estado en territorios que han sido manejados en su mayoría por grupos al margen de la ley: Por lo tanto, este documento busca identificar condiciones en la población al momento de un abandono forzado de sus tierras, partiendo de las condiciones regionales de la población que las hace vulnerables a situaciones de violencia.

Este documento presenta características socioeconómicas y de educación de los hogares en los de- partamentos en los cuales existe un mayor número de personas afectadas por desplazamiento forzado, se realizan estimaciones con variables individuales y departamentales para mostrar como la situación de las personas y de los hogares se afectan por esta condición social.

El documento se divide en cuatro secciones adicionales a esta introducción. En la segunda se realiza una revisión al marco teórico acerca del desplazamiento forzado haciendo énfasis en la propagación de este fenómeno en el país. En la tercera se realiza una caracterización empírica acerca del comportamiento del desplazamiento forzado en Colombia. En la cuarta se presentan las estimaciones realizadas y los principales resultados. Finalmente se encuentra la sección de conclusiones.

\section{Determinantes y Consecuencias del Desplazamiento Forzado}

Algunos estudios muestran que los grupos al margen de la ley persiguen intereses económicos sobre las

\footnotetext{
1 "Es desplazado toda persona que se ha visto forzada a migrar dentro del territorio nacional abandonando su localidad de residencia o actividades económicas habituales, porque su vida, su integridad física, su seguridad o libertad personales han sido vulneradas o se encuentran directamente amenazadas, con ocasión de las siguientes situaciones: Conflicto armado interno, disturbios y tensiones interiores, violencia generalizada, violaciones masivas de los derechos humanos, infracciones al derecho internacional humanitario u otras circunstancias emanadas de las situaciones anteriores que pueden alterar o alteren drásticamente el orden público" (Ley 387 de 1997).
} 
regiones y utilizan acciones para provocar migración de personas hacia otras regiones, donde las situaciones de conflicto armado llevan a que la población se desplace de sus tierras y dichos grupos tomen posesión de las mismas. Situaciones como ataques armados a población civil, toma de rehenes, masacres, asesinatos son causas directas del desplazamiento involuntario de población (Springer, 2006).

Autores como Cuchumbé \& Vargas (2007) hacen referencia a que la expansión territorial por parte de los actores armados y el auge del narcotráfico son las causas que más han intensificado el desplazamiento en Colombia. Al respecto, Ibáñez \& Vélez (2006) argumentan que el secuestro o la extorsión son alternativas de subsistencias por parte de actores armados que les permite expansión, control territorial y explotación de recursos naturales (IDMC, 2006).

González (2002) evidencia que la población huye de su lugar de origen hacia centros con mayor densidad poblacional con el fin de protegerse de las amenazas. Este planteamiento coincide con Ibáñez y Kirchhoff (2001) en la teoría de la nueva economía de la migración donde estudian comportamiento de los agentes frente al riesgo, en donde la supervivencia podría ser un estímulo para diversificar riesgo. (Stark, 1991a, 1991b).

Ibáñez \& Kirchhoff (2001), analizan impactos causados por el desplazamiento forzado en las familias, encontrando que estas modifican su composición, olvidan sus costumbres cuando se adaptan a la cultura del lugar al que migran, generando perdida de propiedades y cambios en sus condiciones de vida. Los niños son los integrantes de la familia más afectados por este proceso, muchos pierden oportunidades de recibir educación, nutrición y atención en salud.

Ibáñez (2006) afirma que gran parte de la población desplazada recurre al mercado informal como medio de subsistencia, llevando a que las familias desplazadas se ubiquen por debajo de la línea de pobreza (OIM ; Ibañez \& Querubin, 2004). Los resultados indican que la situación de los hogares desplazados es dramática, porque en su mayoría son niños, jóvenes y minorías étnicas; se evidencia una tasa alta de deserción escolar, agudas condiciones de pobreza, que en vez de mitigar su situación con la llegada a los lugares de destino se desmejora y cada vez tiende a empeorar.

Actualmente gran parte de los municipios son expulsores de población, según el sistema único de registro, los departamentos con mayor número de población desplazada son Antioquia, Bolívar, Magdalena, Cesar, Putumayo, Caquetá, Meta y Nariño; departamentos que se han caracterizado por ser lugares de intenso conflicto armado y de disputa por los grupos al margen de la ley.

Estos departamentos, presentan regiones con bajas condiciones económicas (Bello, 2004) abundancia en recursos naturales y ser lugares estratégicos para llevar a cabo actividades ilícitas, son extensiones de tierra en las cuales se pueden desarrollar en grandes magnitudes el negocio del narcotráfico, indicando con esto que existen intereses económicos que afectan a esta población. Al analizar el número de hectáreas sembradas de coca y amapola en el 2005, se encuentra que los departamentos con mayor número de estos cultivos son Nariño, Antioquia, Guaviare, Putumayo, Meta y Santander, estos departamentos además de tener altos índices de expulsión de población, tienen grandes magnitudes de tierras destinadas al narcotráfico y a la siembra de cultivos ilícitos, mostrando una relación directa entre dichas variables (Ilustración 1).

\section{Aspectos Socio-Económicos de las Regiones}

En cuanto a la cobertura de salud, indicadores como la tasa de mortalidad del 2005 y el número de afiliados a régimen subsidiado y contributivo, aproximan la presencia del estado en dichas zonas.

Dentro de los datos analizados se encontró que los departamentos con menores tasas de mortalidad infantil fueron Sucre, Nariño, Santander, Casanare, Valle, Tolima y Quindío; en cuanto al número de afiliados al régimen subsidiado los departamentos con mayor cobertura fueron Antioquia, Bogotá, Valle, Cundinamarca, Santander, Bolívar, Atlántico, Nariño y Cesar (Anexo 1). 


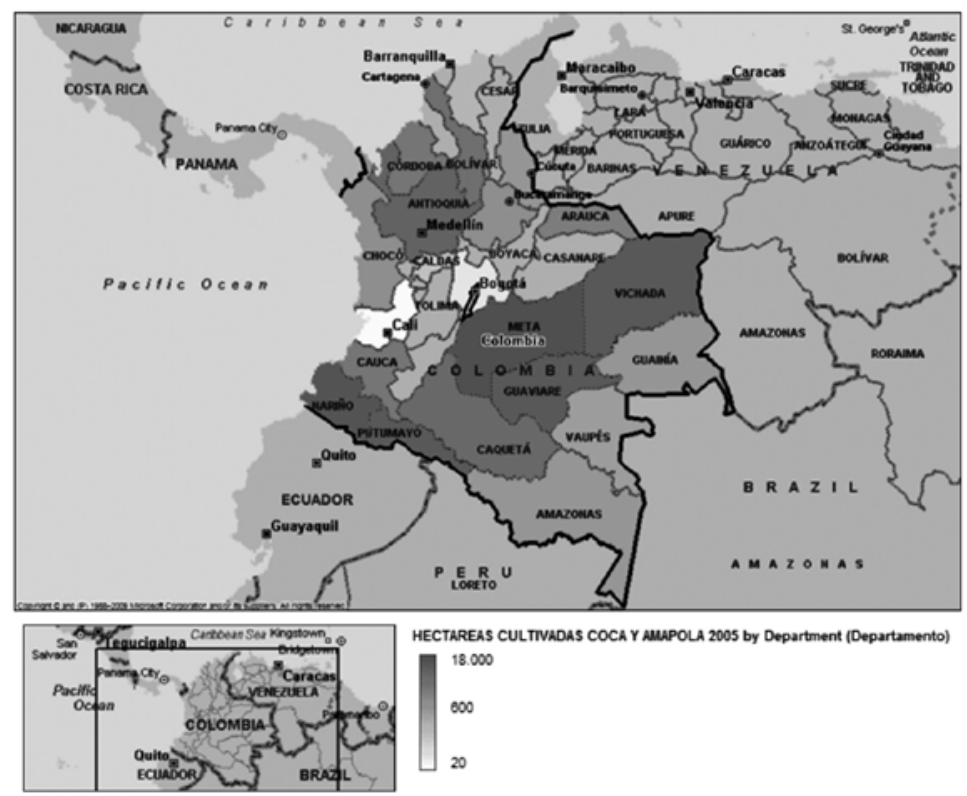

Ilustración 1. Hectáreas Cultivadas Coca y Amapola $2005^{2}$

Respecto a la cobertura en educación, teniendo en cuenta el número de establecimientos oficiales en el 2005 y el número de estudiantes matriculados; Antioquia, Cundinamarca, Santander, Valle, Boyacá, Nariño y Córdoba tienen el mayor número de instituciones educativas instauradas por el Estado; mientras que Bogotá, Antioquia, Valle, Cundinamarca, Bolívar, Atlántico, Santander y Córdoba cuentan con el mayor número de estudiantes matriculados (Anexo 2). Cabe notar que en el caso de los indicadores de cobertura en salud, los departamentos con mayores índices de expulsión tienen índices de mortalidad infantil significativos, por lo que la cobertura en el régimen subsidiado es deficiente de acuerdo a la población del departamento.

Los departamentos expulsores no cuentan con un número adecuado de instituciones, estudiantes matriculados y presentan altos niveles de deserción con pocas instituciones de carácter oficial, mostrando que la cobertura en salud y educación por parte del estado en estas zonas es bastante inferior a la que se tiene en otras regiones del país. Las regalías petroleras y mineras permiten establecer el potencial económico de las regiones; en el caso del carbón los departamentos con mayor número de regalías fueron Cesar, Guajira, Magdalena, Boyacá, Norte de Santander, Atlántico, Antioquia, Córdoba y Bolívar para el año 2007, mostrando que en estas zonas se cuenta con importantes yacimientos de este mineral.

Adicionalmente, los datos muestran que para el 2007 las regalías por concepto de crudo y gas por departamento fueron en mayor proporción para Casanare, Arauca, Meta, Huila, Tolima, Santander, $y$ entre otros Antioquia y Putumayo, mostrando que en estos lugares la exploración y explotación de crudo y gas es mucho más intensa que en las regiones que no reciben regalías o de aquella que las reciben en una proporción muy inferior (Ilustración 2).

2 Fuente: Dane, cálculos de los Autores. 


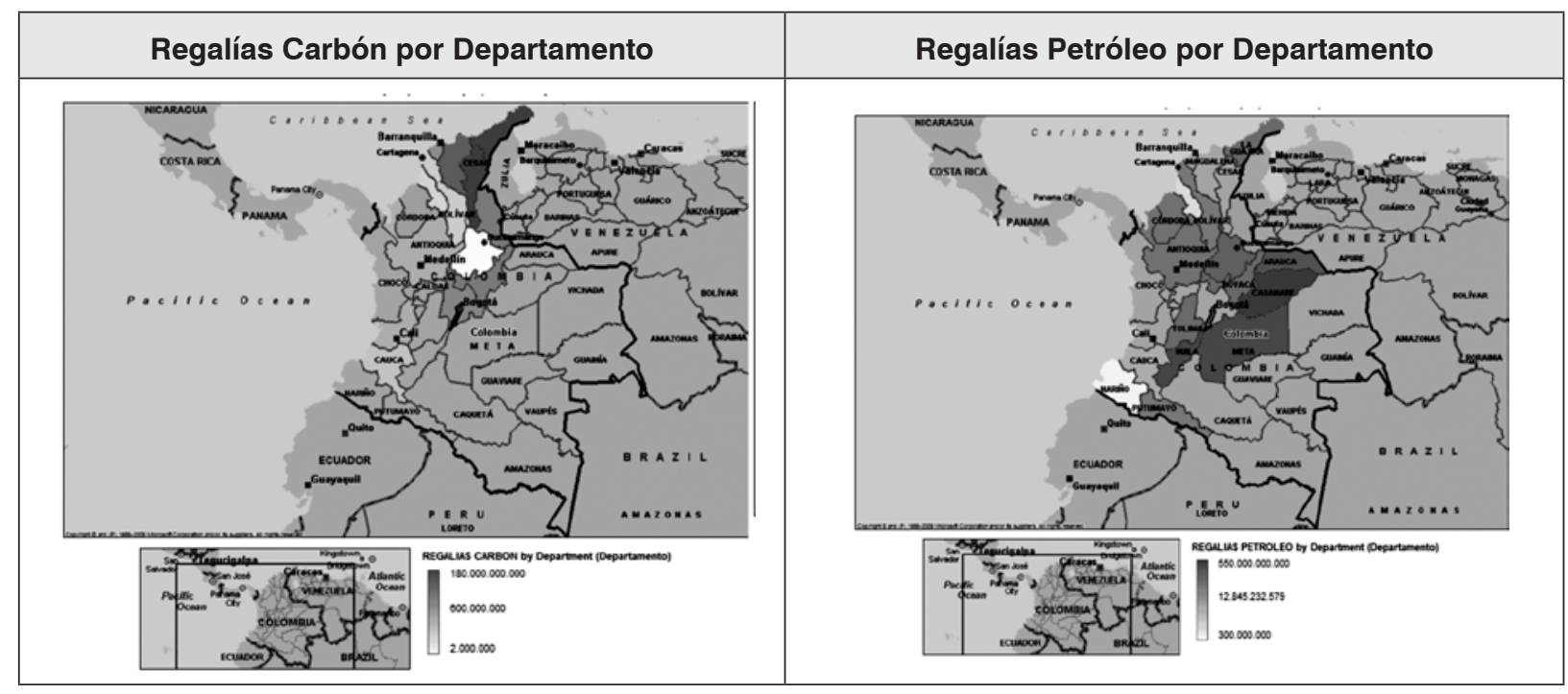

Ilustración 2. Potencial económico - Regalías petroleras y mineras $(2005 \text { - 2007) })^{3}$

Lo anterior, confirma los hallazgos de Pérez (2001) quien indica que el desplazamiento forzado se presenta en territorios ricos en recursos y sobretodo muy estratégicos; ahora siguiendo las cifras presentadas y confrontando las zonas con mayor expulsión de población desplazada, se obtiene que por lo menos 7 de estos 8 departamentos cuentan con un fuerte potencial de recursos naturales y minerales.

Por ello se puede concluir que las regiones expulsoras de población se caracterizan por tener zonas que permiten el desarrollo de cultivos ilícitos, especialmente de coca y amapola, son zonas con alto potencial económico, abundantes en recursos minerales. Así mismo, se nota que son regiones con menor presencia del estado en cuanto a cobertura de servicios de tipo social como educación y salud, factor que influye de manera directa en la vulnerabilidad de dicha población.

\section{Población Desplazada}

A partir de la información encontrada en el censo de población 2005, logro establecerse que existe una mayor concentración de personas desplazadas entre los 0 y 19 años de edad, equivalente al 43\%. En promedio para el total de desplazados hay un niño menor de 1 año por cada 100 mujeres en edad fértil y 28 niños menores de 5 años por cada 100 mujeres en edad fértil. Esta proporción indica que los hogares desplazados llevan consigo menores de edad, lo cual agudiza un poco más la situación de desplazamiento y la hace más complicada en los lugares de destino.

Se encontró que no existe gran diferencia en cuanto a la composición sexual de los desplazados forzados, $50.2 \%$ corresponden a mujeres y $49.8 \%$ son hombres, dicha composición se mantiene para la población en edad de trabajar, $50.8 \%$ y $49.2 \%$ respectivamente.

Ministerio de Minas y Energía, Cálculos de los autores. Las regalías recibidas por recursos naturales en los departamentos, permiten establecer la relación entre desplazamiento y concentración de tierra como medio de obtención de riqueza. El mapa que relaciona regalías de carbón por departamento muestra que la mayoría de los recursos (color oscuro) fueron destinados a Cesar, Guajira, Magdalena, Boyacá, Norte de Santander, Cundinamarca, Atlántico, Antioquia y Valle del Cauca, mientras que el mapa que relaciona regalías por petróleo hace referencia como mayores receptores de dichos recursos a Casanare, Arauca, Meta, Huila, Tolima, Santander, Boyacá y Guajira. 


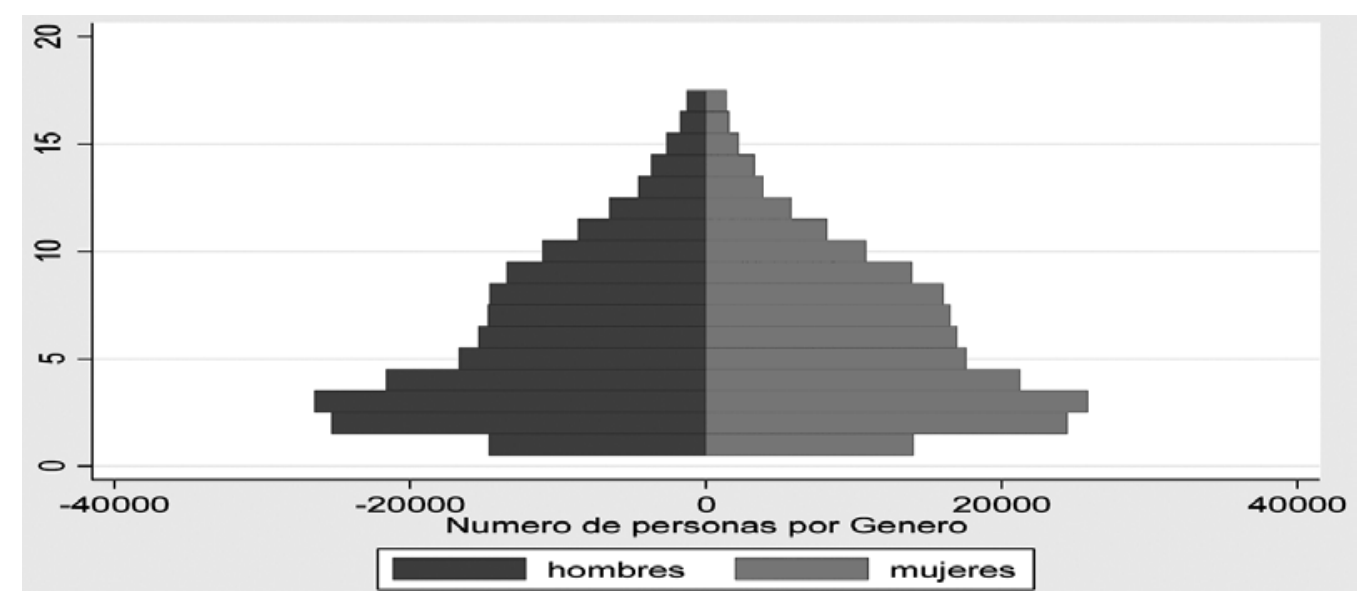

Ilustración 3. Composición Etarea Desplazados Forzados (2005)

Con respecto al estado civil, 34.5\% es casado o está en unión libre y $29.36 \%$ son solteros. La jefatura del hogar es asumida por mujeres lo que hace un poco más complicada la subsistencia en los lugares de destino puesto que estas cabezas de familia son responsables de niños menores de edad. Las tasas de fecundidad para esta población son de 5 niños menores de un año por cada 100 mujeres en edad fértil, cerca de un $4 \%$ de la población desplazada son menores de un año, $8 \%$ menores de 4 años y aproximadamente un 15\% menores de 9 años. Unas de las poblaciones más afectadas con el desplazamiento son las comunidades étnicas, las cuales por su alto grado de vulnerabilidad han sido golpeadas por esto, según acción social el 24\% de desplazados pertenecen a alguna etnia, con mayor participación de la comunidad afrocolombiana, seguido por indígenas y palenqueros.

\subsection{Educación y Desplazamiento Forzado}

Los datos evidencian que el desplazamiento se ha convertido en un foco de pobreza, desigualdad, desempleo e indigencia, gran proporción (61\%) de las personas desplazadas tienen un nivel de educación muy bajo, en su gran mayoría no han terminado el nivel de básica primaria, en razón a que provienen de áreas rurales donde el acceso a la educación es limitado. Un 28\% de dicha población ha cursado algunos años de educación secundaria, lo cual indica que la población desplazada tiene niveles de educación muy inferiores al promedio nacional y por tanto la hace más vulnerables frente a las problemáticas que se desenvuelven en las regiones. Al evaluar la tasa de alfabetización según datos censales, la población desplazada presenta $86 \%$ en promedio frente a $89 \%$ del promedio nacional, mostrando falta de atención estatal en cobertura en educación y es un reflejo de la fragilidad de estos hogares (Ilustración 4).

4 Fuente DANE, cálculo de los autores. Los rangos de edad para la población desplazada se establece de acuerdo a edades quinquenales siendo $1=$ Menores de 4 años, 2=5 a 9 años y así hasta el rango 17 que indica población de más de 80 años. Es importante distinguir que el desplazamiento afecta en mayor proporción a personas con rangos de edades hasta los 24 años. 
Al respecto, Ruiz (2007) determina que la mediana promedio de los años de educación, corresponde a 5,6 años para las mujeres desplazadas y 8,6 para el total de mujeres del país; así mismo muestra que las mujeres en situación de desplazamiento, $11,1 \%$ no sabe leer comparado con un 3,2\% del total nacional, situación que pone en evidencia las desventajas que sufren las mujeres desplazadas. Al respecto, los datos censales evidencian que la población desplazada presenta muy bajos niveles de educación. El Ilustración 5 muestra que dicha población se concentra en niveles de educación Primaria 45.54\%, Secundaria $17.13 \%$, y ningún tipo de educación $12.77 \%$.

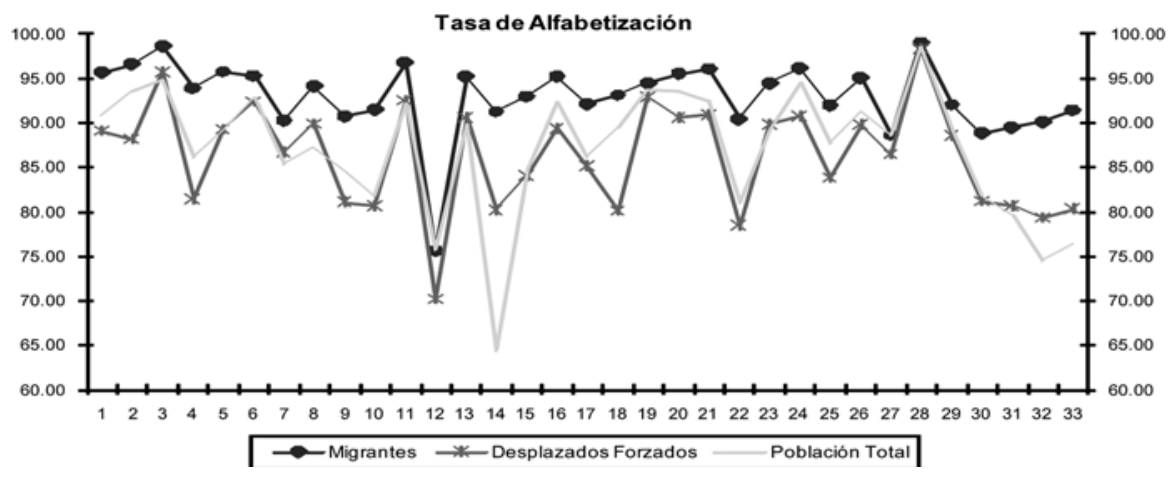

Ilustración 4. Alfabetización Población Total, Migrantes y Desplazados Forzados (2005) ${ }^{5}$

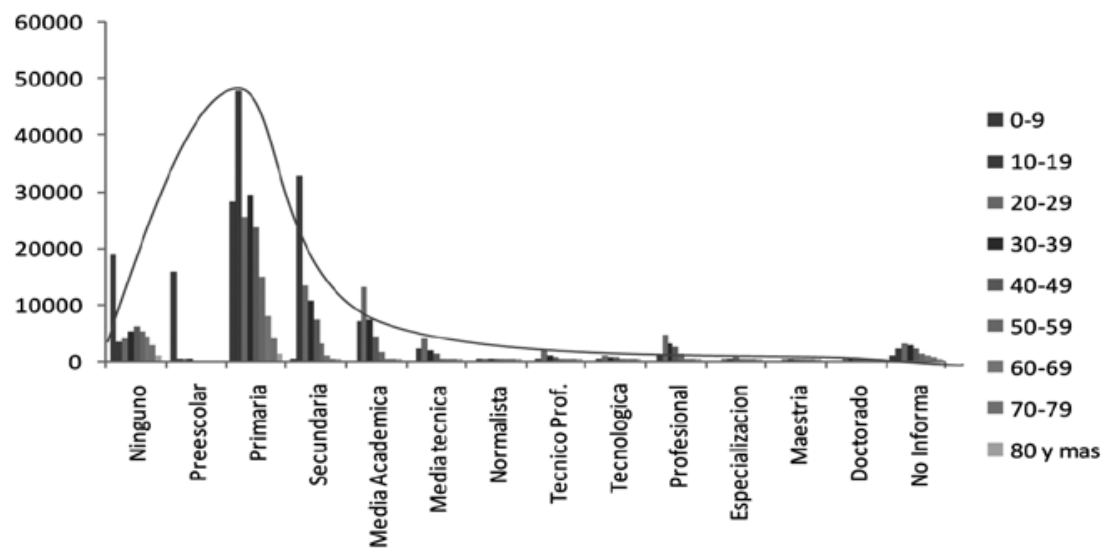

Ilustración 5. Nivel de Estudios por rango de Edad Desplazados Forzados (2005) ${ }^{6}$

\subsection{Inserción laboral de la población desplazada}

En cuanto al desempeño laboral antes y después de la situación de desplazamiento, se encontró que $63.38 \%$ se dedicaba a actividades de agricultura, ga- nadería, caza o silvicultura, seguido por un $9.19 \%$ dedicada a actividades comerciales y un $27.4 \%$ dedicadas a actividades de servicios, la construcción, etc. Esta información la corrobora el sistema de información sobre población desplazada por la vio-

\footnotetext{
5 Fuente DANE, Cálculos de los autores. (Ver relación departamentos Anexo 3).

6 Fuente DANE, Cálculos de los autores.
} 
lencia en Colombia, el cual registra que antes del desplazamiento las personas que desarrollaban algún tipo de actividad económica 35.61\% lo hacían en la agricultura, un $26.23 \%$ realizaban trabajos propios del hogar y el restante se desempeñaban en oficios varios.

Esta situación cambio luego de tener que desplazarse de manera forzada hacia ciudades o centros urbanos en los cuales un $42.82 \%$ de esta misma población no ha podido vincularse a ninguna actividad de tipo laboral, $13.56 \%$ desarrolla trabajos informales, $11.86 \%$ labores propias del hogar y el restante se dedica a otro tipo de actividades que les significan un mínimo ingreso para subsistir.

Con respecto a la inserción en el mercado laboral siguiendo el censo 2005 (Anexo 5), se identifico que $71 \%$ de la población se encuentra en edad de trabajar, y la población económicamente activa asciende apenas a un 32\%; el índice de desempleo es muy superior al nacional, en promedio $12 \%$ para el total de dicha población, siendo Bogotá el principal destino de los desplazados con 18\% aproximadamente.

Según Ruiz (2007), 38.3\% de las mujeres entre 13 y 49 años estaban trabajando, contra un $51.6 \%$ del total de mujeres del país; cabe anotar que el desempeño laboral para la población desplazada se refiere a ventas informales y servicios. El empleo informal y el subempleo están siendo considerados dentro de las tasas de ocupación, debido a que la población desplazada considera el mercado informal para subsistir dentro de la nueva ciudad de destino.

Finalmente a partir de los datos censales, las tasas de dependencia de la población desplazada ascienden a $5.22 \%$, del total de la población en edad de trabajar solo un $32 \%$ se encuentra dentro de la población económicamente activa y de esta ultima $88 \%$ tiene algún tipo de vinculación laboral, por lo cual la dependencia de tipo económico es muy superior al nacional.

\subsection{Desplazamiento y entorno familiar}

Según los cálculos hechos con datos censales, los hogares desplazados tienen al menos una necesidad básica insatisfecha, por tanto se encuentran por debajo de la línea de pobreza luego del desplazamiento ${ }^{7}$. Un $40 \%$ de los hogares desplazados habita en viviendas inadecuadas no solo por las condiciones físicas sino por los niveles de hacinamiento a los que se ven sometidos, gran proporción de esta población vive en habitaciones alquiladas en las que se acomodan independientemente de sus necesidades.

Cerca de un $46 \%$ de los hogares desplazados no tienen acceso a condiciones mínimas de tipo sanitario, no cuentan con un sanitario propio en su hogar y no tienen acceso a servicios públicos de acueducto y energía. Tal población antes del desplazamiento vivía en condiciones económicas y sociales que les permitían subsistir en su medio y acceder a bienes y servicios de los que han sido privados luego de su situación de desplazamiento; por ello la pobreza e indigencia de gran parte de la población desplazada obedece a situaciones que han tenido que enfrentarse producto del desplazamiento.

Las condiciones de pobreza y miseria obedecen al bajo acceso que tienen a la sociedad y al mercado laboral, explicado por la condición de desplazados que no les permite acceder a este tipo de mercados debido a que muchos se encuentran indocumentados, por otro lado la población se encuentra llena de miedo y amenazas por lo que no accede a entes delegados por el estado para recibir la ayuda necesaria por temor a ser encontrados; gran parte de la población desplazada ha sido enfrentada a centro urbanos en los que son vulnerables y su nivel educativo o su experiencia laboral no es suficiente a la hora de acceder al mercado laboral.

Por lo tanto, a pesar de los esfuerzos estatales por mantener programas que permitan a la población víctima del desplazamiento reintegrarse a la sociedad y obtener ayudas, es necesario políticas de lar-

7 Aproximadamente un 48\% de dichos hogares tienen 3 o más necesidades básicas insatisfechas, situación que refleja las agudas condiciones económicas en las cuales habita dicha población y los grandes niveles de indigencia a los que se ven enfrentados. 
go plazo que solucionen problemáticas coyunturales para estabilizar condiciones de los desplazados, mejorando calidad de vida, capacidad productiva y protección Estatal.

\section{Zonas de expulsión y conflicto}

Ibáñez \& Querubín (2004), atribuyen el fenómeno del desplazamiento forzado a la aguda situación de conflicto armado que presentan las regiones, por cuenta de homicidios y enfrentamientos entre grupos al margen de la ley con las fuerzas armadas; mientras que acción social durante 1997-2007 informó que en promedio $60 \%$ de la población desplazada atribuye como autor de desplazamiento a las autodefensas, paramilitares y guerrillas (50\%), seguido por otros grupos insurgentes no identificados (24\%); estos grupos se ven motivados a desplazar población de forma masiva por la apropiación de tierras y regiones que les permitan desarrollo de actividades ilícitas o la explotación de recursos para financiar la lucha con el estado.

Según datos de la Policía Nacional, los departamentos con más acciones subversivas fueron Nariño, Cauca, Putumayo, Guaviare, Arauca, Antioquia y Valle; casos como Putumayo, Arauca, Antioquia Meta y Norte de Santander, donde se presentaron el mayor número de actos terroristas a su vez son los departamentos expulsores de desplazados.
Por otra parte, teniendo en cuenta delitos cometidos por departamento, se identifica que en los 33 departamentos y 5 áreas metropolitanas los delitos más frecuentes a nivel nacional es el hurto a personas seguido por el hurto a residencias (Sandoval et al., 2009). La media de homicidios comunes es de 2925 considerando 5 años, los departamentos que superan este promedio son Antioquia, Atlántico, Bolívar, Boyacá, Caldas, Caquetá, Cauca, Cesar, Córdoba, Cundinamarca, Choco, Huila, La Guajira, Magdalena, Meta, Nariño, N. Santander, Quindío, Risaralda, Santander, Sucre, Tolima, Antioquia, Bogotá D.C. y Valle del Cauca siendo los tres últimos los que tienen los niveles más altos de homicidio.

Los delitos son clasificados entre los que afectan la seguridad democrática y los que afectan la seguridad ciudadana. El secuestro, la extorsión y el terrorismo son delitos que se relacionan con la presencia de conflicto en Colombia que afectan directamente la seguridad nacional. El terrorismo, es el principal método que utilizan las organizaciones armadas para desestabilizar democracia y según la constitución constituye un delito tipificado. Los departamentos en donde se encuentran delitos relacionados con terrorismo son Meta, Norte de Santander, Putumayo, Huila, Antioquía y Arauca siendo los dos últimos los que tienen niveles más altos.

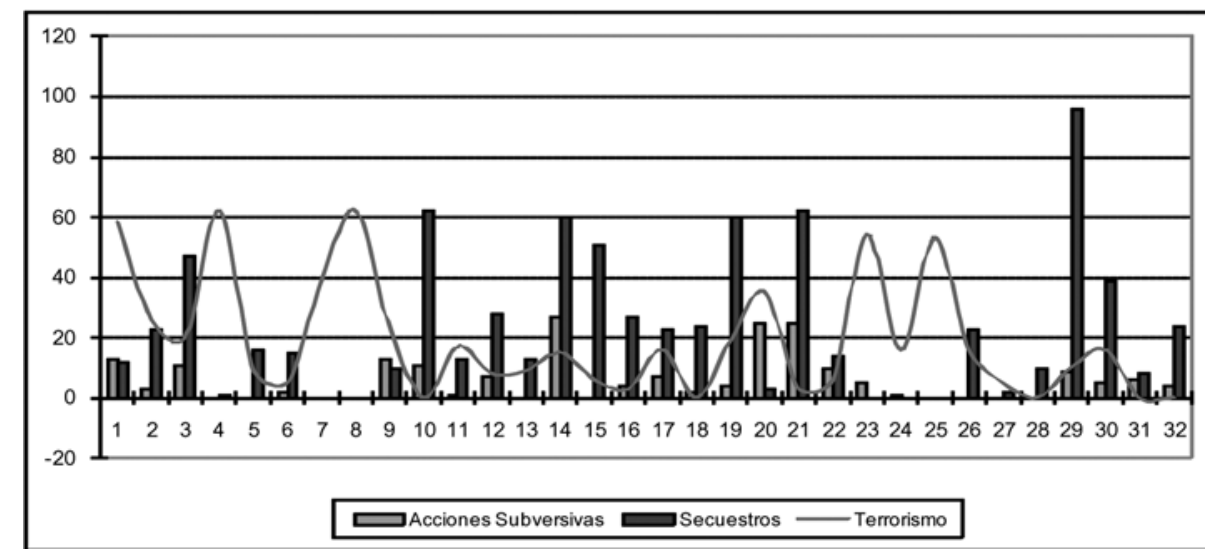

Ilustración 6. Acciones Subversivas, Secuestros y Terrorismo en Colombia (2005) ${ }^{8}$

8 Fuente: Policía Nacional, DNP, Cálculos de los Autores. (Anexo 2 Relación Departamentos). 
La ocurrencia de delitos de secuestro y extorsión tienen características similares ya que el mayor número de delitos se encuentran en Antioquia, Atlántico, Caldas, Cesar, Cundinamarca, Magdalena, Meta, Nariño, Norte de Santander, Risaralda, Santander, Sucre, Tolima, Huila, Bucaramanga, Cali, Medellín y Bogotá siendo esta última la ciudad con mayor número de extorsiones. Con respecto a los departamentos receptores de migrantes forzados, Cundinamarca, Bolívar, Antioquia, Santander, Valle del Cauca, Norte de Santander y Córdoba, absorbieron el 65\% de los desplazados.

Según el Censo 2005, dentro de los departamentos receptores se encontró que Bogotá alberga 20\% del total de la población desplazada, seguido por Valle $(9,27 \%)$, Cundinamarca $(7,94 \%)$, Huila $(5,20 \%)$ y Antioquia (4,97\%); lo cual reafirma que las capitales son el foco de atracción de desplazados y en general de migrantes internos. Estas cifras representan concentración de esta población en los centros de desarrollo del País. En cuanto a los departamentos expulsores, los departamentos con mayor expulsión de desplazados son Antioquia (8,14\%), Caquetá (7,43\%), Tolima (7,20\%), Putumayo $(5,81 \%)$, Bolívar (4,91\%), Cesar (4,81\%) y Valle $(4,47 \%)$; en estos departamentos se desarrollaron cerca del 36\% de los actos terroristas y el $26 \%$ de homicidios registrados en el periodo.

La población desplazada y que migra interdepartamentalmente, lo hace con mayor frecuencia de áreas rurales a urbanas ya que se tiene la percepción que en dichos centros existe mayor facilidad de reubicación y acceso a oportunidades de tipo laboral, lo cual ha fortalecido el proceso de urbanización del país. Adicionalmente, esto tiene impactos sociales en la medida en que la población desplazada incrementa mano de obra en las grandes ciudades causando más desempleo, más pobreza y mayores índices delincuenciales.

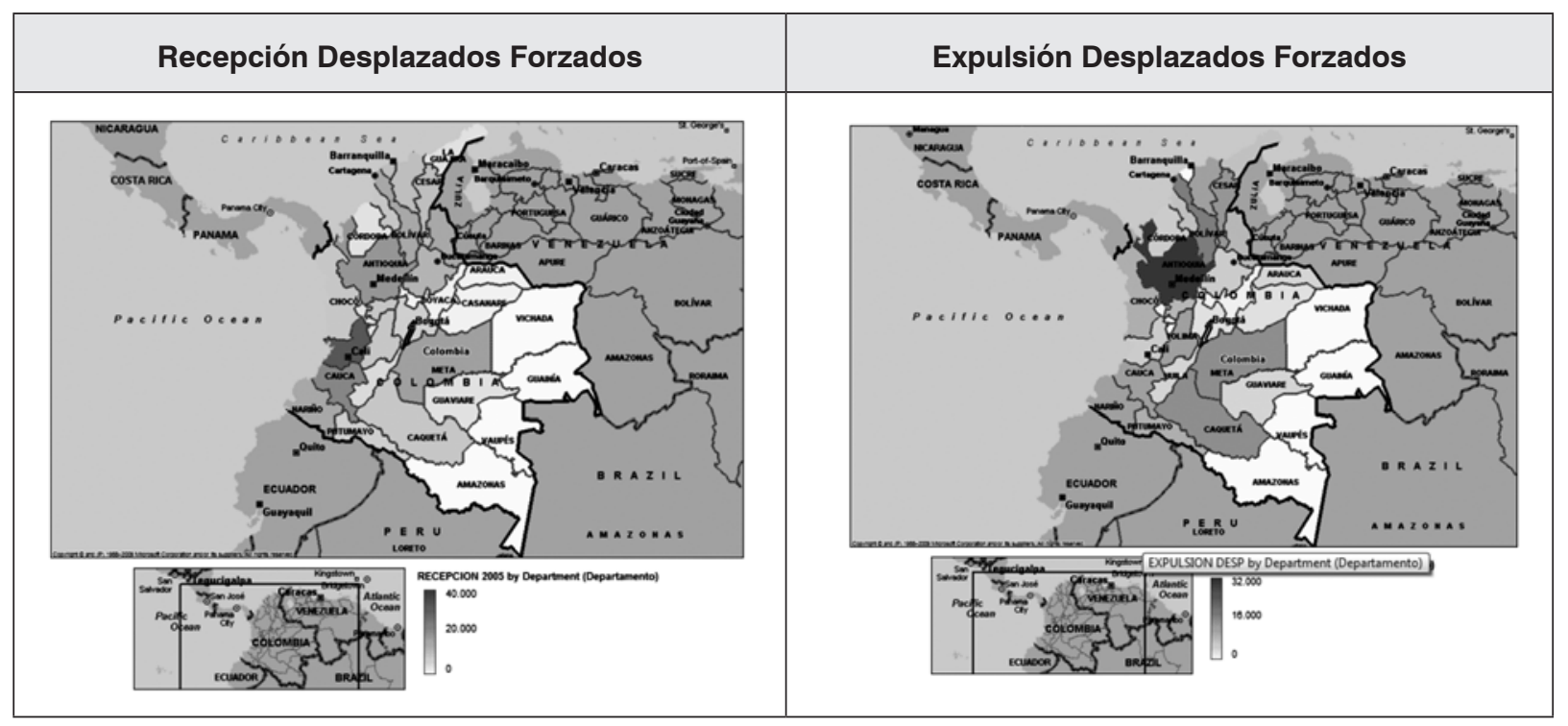

Ilustración 7. Zonas de Recepción y Expulsión de Desplazados Forzados. Censo (2005)9

9 Fuente: DANE, Cálculos de los autores. 
Por esta razón, la siguiente sección pretende mostrar que el desplazamiento forzado no solamente obedece a las características de violencia y conflicto presentes en los departamentos, sino que obedece a condiciones económicas que aumentan desigualdad departamental, así como a las características de educación básica en los departamentos que motiva aumentos en el nivel de desplazamiento hacia regiones con mayores oportunidades de educación.

\section{Metodología de análisis}

Los estudios sobre desplazamiento forzado apuntan como principales determinantes a variables asociadas con conflicto armado y violencia municipal, por ello además de tener en cuenta dichas variables, se complementará el análisis con variables de educación presentes en los departamentos, así como variables de desigualdad y nivel socio económico departamental que permitan mostrar como el desplazamiento forzado no solo depende de las condiciones de violencia regional, sino además de las características de educación y desigualdad propias de las regiones.

Por lo tanto, para encontrar variables que expliquen el aumento en el desplazamiento forzado a nivel departamental vinculado a educación, vulnerabilidad económica y violencia departamental, inicialmente se realizarán estimaciones lineales para conocer el signo de las variables relevantes que posteriormente con estimaciones binarias se podrá estimar la decisión de desplazamiento controlando por características departamentales de educación, nivel socioeconómico y violencia regional para el año 2005. Posteriormente, a partir de la significancia observada en las variables se estima un panel de datos para los años 2003, 2004, 2005, mediante el cual se puedan controlar características no observables por departamento.

$10 \mathrm{TDF}_{\text {norm }}=\frac{\left(\frac{\mathrm{E}_{\text {norm }}}{\mathrm{PT}_{\text {norm }}}\right)}{10}$.

$\mathrm{E}_{\text {norm }}$ : Expulsados Normalizados; $\mathrm{PT}_{\text {norm }}$ : Población Normalizada

\subsection{Estimaciones lineales}

Para establecer la relación de la tasa de desplazamiento forzado (TDF) en los departamentos del país, a partir de condiciones de educación, nivel socioeconómico y violencia departamental, se realizan estimaciones individuales con variables que permitan relacionar las condiciones del departamento con el nivel de desplazamiento regional.

La TDF ${ }^{10}$, se define a partir del coeficiente que relaciona el número de personas expulsadas y la población por departamento, estas variables fueron normalizadas de la forma como se presenta en el apéndice con la intención de tener variables en un intervalo continuo para evitar signos negativos que pudieran alterar los resultados.

La estimación inicial (Anexo 6), tiene en cuenta establecimientos de primaria, secundaria, alumnos y docentes que permitan explicar la variación en la migración interna. Las variables son ept: establecimientos de primaria totales; epo: establecimientos de primaria oficiales; epno: establecimientos de primaria no oficiales; apt: alumnos de primaria totales; apo: alumnos de primaria establecimientos oficiales; apno: alumnos de primaria establecimientos no oficiales; eso: establecimientos de secundaria oficiales; esno: establecimientos de secundaria no oficiales; aso: alumnos de secundaria establecimientos oficiales; asno: alumnos de secundaria establecimientos no oficiales; dso: docentes de secundaria establecimientos oficiales; dsno: docentes de secundaria establecimientos no oficiales.

$T D F=\alpha+\beta E P T_{i}+\rho A P T_{i}+\varepsilon E S T_{i}+\sigma A S T_{i}+\vartheta D E S E_{i}+\mu$

Los resultados, muestran que el número de estudiantes de primaria de establecimientos oficiales y totales, el número de establecimientos de secun- 
daria oficiales y el número de docentes de establecimientos no oficiales, inciden en la variación del desplazamiento a nivel departamental.

La siguiente estimación (Anexo 7), tiene en cuenta condición económica, condiciones de vida y tipo de población para explicar la variación en la migración interna. Las variables son nbi: necesidades básicas insatisfechas; tmort05: tasa de mortalidad; irnorm: índice de rotación normalizado; ipnorm: índice de presión normalizado; desempleo05: desempleo departamental.

$$
T D F=\alpha+\beta N B I_{i}+\rho M O R T_{i}+\varepsilon I R_{i}+\sigma I P_{i}+\vartheta D E S E_{i}+\mu
$$

En este caso, tan solo el índice de rotación y el desempleo departamental, inciden en la variación del desplazamiento; siendo importante para que en el análisis posterior se utilicen mayor número de observaciones para armar un análisis consistente en torno a las características económicas y el nivel de desplazamiento. Por su parte, la siguiente estimación (Anexo 6) tiene en cuenta variables de violencia y conflicto, así como de presencia del estado en los departamentos, con las cuales se pueda explicar la variación en la migración interna. Las variables son homcom: homicidios comunes; secuest: secuestros; extors: extorsiones; terror: acciones terroristas; hres: hurto a residencias; hcom: hurto comercio; hauto: hurto automotores; ep: estaciones de policía.

$$
\begin{aligned}
& \text { TDF }=\alpha+\beta \text { HCOM }_{i}+\rho \text { SECUES }_{i}+\varepsilon \text { HURTOS }_{i}+ \\
& \vartheta E P_{i}+\mu
\end{aligned}
$$

Los resultados, muestran que los secuestros, acciones terroristas y la presencia de estaciones de policía en los departamentos, inciden en la variación del desplazamiento; siendo importante para mostrar que las características de violencia y la baja presencia del estado en las regiones por medio de estaciones de policía, provoca alteraciones en las migraciones internas.
Por su parte, la estimación probit (Anexo 6) pretende mostrar como en los departamentos la decisión de desplazamiento obedece a las características de educación, población y violencia interna que motiva la probabilidad de migrar de un departamento hacia otro, el cual se realiza teniendo en cuenta las variables que resultaron significativas en las estimaciones anteriores.

\subsection{Estimación Probit}

El modelo probit, define una variable aleatoria binaria que puede tomar valores de 0 y 1 , teniendo en cuenta una muestra aleatoria de n-observaciones de la variable aleatoria, tal que existe un conjunto de vectores con información relevante que explican el comportamiento de la variable aleatoria. La variable dependiente ( $\mathrm{dtdf}$ ) toma valores de alto $^{11}$ nivel de desplazamiento (1) ó bajo ${ }^{12}$ nivel de desplazamiento (0), a partir de unas variables independientes cuantitativas de educación, nivel de población y condiciones de violencia; en este caso el modelo probit busca obtener una combinación lineal de las variables independientes que permita calcular la probabilidad de respuesta de la variable dependiente frente a los diferentes estímulos o variables independientes.

Dados los coeficientes, se puede afirmar que variaciones en establecimientos de primaria no oficiales, alumnos de primaria oficiales, establecimientos de secundaria oficiales y no oficiales, así como la rotación de la población y las acciones terroristas, aumentarían la probabilidad en el desplazamiento forzado a nivel departamental, mostrando que no solo las condiciones de violencia y conflicto interno determinan el desplazamiento forzado, sino a su vez la oferta de establecimientos educativos presentes en los departamentos y la rotación de las personas hacia centros urbanos con mayores condiciones económicas motiva migraciones al interior del país.

Las estimaciones anteriores, sirvieron de base para conocer la significancia de las variables en torno a la variable dependiente a pesar de estar limitadas

11 Es alto, si el valor del idf es mayor a 0.1.

12 Es bajo, si el valor del idf es menor a 0.1. 
en el número de variables existentes y en realizar el análisis para un solo periodo de tiempo, por esta razón se pretende realizar un análisis con panel de datos para los años 2003, 2004, 2005 teniendo en cuenta las variables que resultaron significativas en la revisión anterior para tener medidas consistentes en diferentes periodos de tiempo.

\subsection{Estimación por panel de datos}

Las variables empleadas en la estimación (Anexo 7) son variables de educación (epo, epno, apo, apno, eso, esno, aso, asno), población (rae, papib, pob), violencia departamental (homcom, secuest, extors, terror) de manera que permitan establecer la relación con la tasa de desplazamiento forzado. El análisis se inicia estimando el modelo subyacente por mínimos cuadrados ordinarios luego de combinar los datos de corte transversal con los datos de series de tiempo. Posteriormente, se identifican variables que deben ser omitidas porque no contribuyen a la significancia de la estimación, sin ignorar que tales variables pueden conducir a cambios en los interceptos del corte trasversal y las series de tiempo.

Los resultados muestran que la oferta de establecimientos de educación primaria y secundaria, tienen relación con el número de alumnos de establecimientos oficiales y no oficiales, lo cual se relaciona con la tasa de desplazamiento forzado siendo importante para mostrar que las condiciones de educación de los departamentos tienen relación con los incentivos que puedan tener las personas de migrar hacia otras zonas donde puedan tener mejores niveles educativos.

Por su parte, los recursos que brinde el estado a los departamentos, la participación del departamento en el producto interno bruto y la población departamental, inciden en el desplazamiento, siendo importante para mostrar que las condiciones económicas de los departamentos condiciona la decisión de migración interna de las personas por cuanto regiones con mayores posibilidades de desarrollo reduce la desigualdad social y motiva la permanencia en las regiones.

Los homicidios comunes, secuestros, extorsiones y acciones terroristas, en conjunto tienen relación con el desplazamiento forzado en los departamentos, lo cual se sustenta en la literatura revisada previamente, donde se muestra que las características de violencia y conflicto interno de los departamentos, motivan decisiones de desplazamiento, siendo los departamentos con mayores niveles de violencia los que se caracterizan por ser expulsores (gráfico 8) y aquellos con menores niveles de violencia los receptores de personas desplazadas por conflicto armado en sus respectivas regiones de origen.

De esta manera, se ha podido establecer que no solo las condiciones de violencia de los departamentos motivan las decisiones de desplazamiento, sino que son las características económicas de los departamentos y en especial el entorno educativo de las zonas dado por la cantidad de establecimientos educativos que permite a más personas acceder a educación primaria y secundaria, puesto que son los niños y los jóvenes los más perjudicados cuando las familias deciden desplazarse hacia otras regiones del país, en especial hacia ciudades más grandes donde pueden encontrar mayores posibilidades ante las circunstancias de violencia que viven en sus regiones de origen.

\section{Comentarios finales}

Las estimaciones indican que las condiciones de educación y desigualdad económica motivan migraciones internas y fortalecen a los grupos que usan acciones violentas con fines ilícitos, departamentos como Antioquia, Nariño, Cauca, Putumayo, Meta y Valle son los que presentan un mayor número de expresiones de conflicto armado medidas por acciones subversivas, terrorismo y secuestros.

En cuanto a la población desplazada se encontró una concentración de menores de edad (42\%), en promedio existen 27,5 niños por cada 100 mujeres; este grupo etáreo es uno de los más vulnerables y con mayor riesgo a padecer un alto deterioro de su bienestar. Los resultados sobre posibilidades de educación primaria y secundaria son relevantes para explicar la importancia de brindar a los niños y jóvenes de zonas vulnerables mayores posibilidades de acceder a educación básica. 
Al respecto, al analizar los niveles de educación de la población desplazada se encontró que en su gran mayoría tienen un grado de educación muy bajo Primaria $45.54 \%$, Secundaria $17.13 \%$, y ningún tipo de educación $12.77 \%$ lo cual evidencia las deficiencias respecto al cubrimiento y acceso a educación departamental.

Las variables de violencia regional no son las únicas variables que determinan las decisiones de desplazamiento individual, en cambio las condiciones de educación y la atención del estado en cuanto a asignación de recursos juegan un papel importante en el proceso de desplazamiento ya que aquellas regiones que brindan estabilidad económica y social puede reducir incentivos de migrar hacia regiones con mayores oportunidades educativas y económicas.

\section{Referencias}

Bello, M. (2004). El Desplazamiento Forzado en Colombia: Acumulación de Capital y Exclusión Social. En: Revista Aportes Andinos: Globalización, migración y derechos humanos. (7):1-8.

CODHES. (1999). Desplazamiento sin tregua. En: Boletín Consultoría para los Derechos Humanos y el Desplazamiento. $1-3$

Cuchumbé, N. \& Vargas, J. (2007). Reflexiones Sobre el Sentido y Génesis del Desplazamiento Forzado en Colombia. En: Universitas Humanística. (65): 173-196.

González, M. (2002). Desterrados: El desplazamiento forzado sigue aumentando en Colombia. En: Convergencia, Universidad Autónoma de México, Enero-Abril, 27(9): 41-78.
Ibáñez, A. (2006). La Estabilización Económica de la Población Desplazada. En: Working Papers Fundación Ideas para la Paz. No. 3: 1-34

Ibañez, A. \& Kirchhoff (2001). Displacement Due to Violence in Colombia: Determinants and Consequences at the Household Level. Discussion Papers on Development Policy, No. 41

Ibañez, A. \& Querubin, P. (2004). Acceso a tierras y desplazamiento forzado en Colombia. Documentos CEDE, Universidad de los Andes. No. 23: 1-114.

Ibáñez, A. \& Vélez, C. (2003a). Instrumentos de atención de la población desplazada en Colombia: Una distribución desigual de las responsabilidades municipales. En: Documentos CEDE, Universidad de los Andes. No. 37: 1-34.

IDMC . (2006). Internal Displacement: Global Overview of Trends and Developments in 2005. Ginebra: Internal Displacement Monitoring Centre. Norwegian Refugee Council.

Mooney, E. (2005). The Concept of Internal Displacement and the Case for Internally Displaced Persons as a Category of Concern. En: Refugee Survey Quarterly. 24, (3): 9-24.

Pérez, L. (2001). Una Mirada Empírica a los Determinantes del Desplazamiento Forzado en Colombia. En: Cuadernos de Economía. 20(35): 205-243.

Ruiz, N. (2007). El Desplazamiento Forzado en el Interior de Colombia: Caracterización Sociodemográfica y pautas de Distribución territorial 2000-2004. En: Centro de Estudios Demográficos.

Springer, N. (2006). Colombia: Desplazamiento Interno - Políticas Y Problemas. En: Revista Unhcr Writenet. 62.

Stark, O. (1991). The Migration of Labor. En: Economic Development: Harlow: Addison Wesley.

Sandoval et.al (2009). Pobreza y delincuencia departamental en Colombia 2003-2007. En: Revista Facultad de Ciencias Económicas. Investigación y Reflexión. 17(1): 95-108.

Steele, A. (2007). Massive Civilian Displacement in Civil War: Assessing Variation in Colombia. En: The Institute of Development Studies. 


\section{ANEXOS}

\section{Anexo 1. Nivel de educación por tramo de edad}

\begin{tabular}{|c|c|c|c|c|c|c|c|c|c|c|c|c|c|c|}
\hline Edad & № & Free & Frim & Sec & $\begin{array}{l}\text { Me- } \\
\text { dada }\end{array}$ & $\begin{array}{l}\text { Me- } \\
\text { diec }\end{array}$ & Nom & $\begin{array}{l}\text { Tec- } \\
\text { Pr }\end{array}$ & Tec & Pro & Esp & Mae & Doc & NI \\
\hline $0-9$ & 18837 & 15969 & 28109 & 12 & 0 & 0 & 0 & 0 & 0 & 0 & 0 & 0 & 0 & 926 \\
\hline $10-19$ & 3392 & 453 & 47716 & 32646 & 7178 & 2109 & 68 & 404 & 205 & 92 & 0 & 0 & 0 & 2140 \\
\hline $20-29$ & 4141 & 80 & 25572 & 13368 & 13044 & 3927 & 176 & 1620 & 1036 & 4487 & 218 & 52 & 44 & 3012 \\
\hline $30-39$ & 5239 & 9 & 29551 & 10693 & 7402 & 1850 & 131 & 1024 & 793 & 3160 & 546 & 122 & 38 & 2935 \\
\hline $40-49$ & 6015 & 0 & 23780 & 7478 & 4465 & 1185 & 90 & 736 & 597 & 2524 & 649 & 143 & 38 & 2322 \\
\hline $50-59$ & 5288 & 0 & 15030 & 3108 & 1515 & 421 & 79 & 301 & 203 & 1294 & 402 & 122 & 90 & 1444 \\
\hline $60-69$ & 4431 & 0 & 7871 & 955 & 430 & 126 & 55 & 102 & 63 & 357 & 106 & 41 & 22 & 955 \\
\hline $70-79$ & 2817 & 0 & 3967 & 428 & 163 & 70 & 15 & 28 & 15 & 140 & 22 & 9 & 10 & 567 \\
\hline 80 y más & 1103 & 0 & 1235 & 96 & 40 & 12 & 14 & 11 & 9 & 24 & 5 & 1 & 5 & 177 \\
\hline
\end{tabular}

FUENTE: DANE, Cálculos de los autores.

N: Ninguno, Pree: Preescolar, Prim: Primaria, Sec: Secundaria, Medaca: Media Académica, Medtec: Media Técnica, Norm: Normalista, TecPr: Técnico Profesional, Tec: Tecnológica, Pro: Profesional, Esp: Especialización, Mae: Maestría, Doc: Doctorado, NI: No Informa.

Anexo 2. Población desplazada por género

\begin{tabular}{|c|r|r|c|}
\hline \multirow{2}{*}{ Edadedes quinquenales } & \multicolumn{2}{|c|}{ Género } & \multirow{2}{*}{ Total } \\
\hline & Hombre & Mujer & \\
\hline $0-9$ & 40.169 & 38.678 & 78.847 \\
\hline $10-19$ & 49.626 & 47.644 & 97.270 \\
\hline $20-29$ & 36.143 & 48.834 & 70.977 \\
\hline $30-39$ & 30.820 & 32.673 & 63.493 \\
\hline $40-49$ & 25.205 & 24.817 & 50.022 \\
\hline $50-59$ & 15.360 & 13.937 & 29.297 \\
\hline $60-69$ & 8.367 & 7.179 & 15.546 \\
\hline $70-79$ & 4.410 & 3.841 & 8.251 \\
\hline Más 80 & 1.324 & 1.408 & 2.732 \\
\hline TOTAL & 211.424 & 205.011 & 416.453 \\
\hline
\end{tabular}

FUENTE: DANE, Cálculos de los autores. 
Anexo 3. Estimación 1: Educación departamental 2005

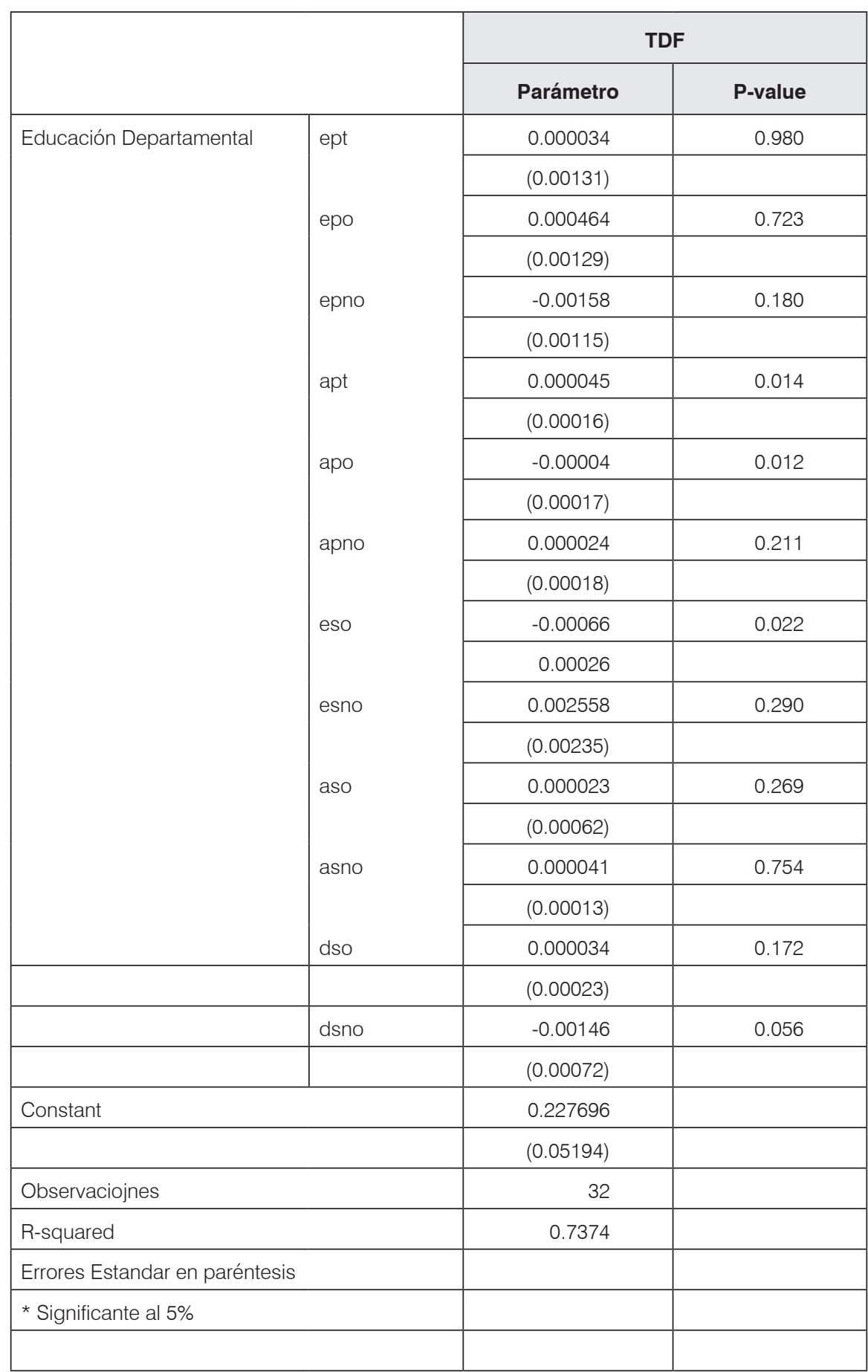


Anexo 4. Estimación 2: Condición departamental 2005

\begin{tabular}{|c|c|c|c|}
\hline & & & \\
\hline & & Parámetros & P-vlue \\
\hline Condición Departamental & nbi & 0.003098 & 0.081 \\
\hline & & $(0.00225)$ & \\
\hline & tmort 05 & 0.001246 & 0.482 \\
\hline & & $(0.006180$ & \\
\hline & imom & -0.01696 & 0.070 \\
\hline & & $(0.01202)$ & \\
\hline & ipnom & 0.013075 & 0.211 \\
\hline & & $(0.01020)$ & \\
\hline & desempleo 05 & 0.0036560 .073 & \\
\hline & & $(0.00639)$ & \\
\hline Constant & & 0.018221 & \\
\hline & & $(0.15263)$ & \\
\hline Observations & & 32 & \\
\hline R-squared & & 0.5714 & \\
\hline Errores Estandar en parént & & & \\
\hline * Significancia al 5\% & & & \\
\hline
\end{tabular}


Anexo 5. Estimación 3: Violencia departamental 2005

\begin{tabular}{|c|c|c|c|}
\hline & \multicolumn{3}{|c|}{ TDF } \\
\hline & & Parámetro & P-value \\
\hline \multirow[t]{16}{*}{ Violencia Departamental } & \multirow[t]{2}{*}{ homcom } & 0.000016 & 0.891 \\
\hline & & $(0.00012)$ & \\
\hline & \multirow[t]{2}{*}{ secuest } & 0.002768 & 0.095 \\
\hline & & $(0.00158)$ & \\
\hline & \multirow[t]{2}{*}{ extors } & -0.00118 & 0.135 \\
\hline & & $(0.00076)$ & \\
\hline & \multirow[t]{2}{*}{ terror } & 0.003526 & 0.020 \\
\hline & & $(0.00141)$ & \\
\hline & \multirow[t]{2}{*}{ hres } & 0.000106 & 0.616 \\
\hline & & $(0.00020)$ & \\
\hline & \multirow[t]{2}{*}{ hcom } & -0.00012 & 0.568 \\
\hline & & $(0.00020)$ & \\
\hline & \multirow[t]{2}{*}{ hauto } & 0.000041 & 0.781 \\
\hline & & $(0.00014)$ & \\
\hline & \multirow[t]{2}{*}{ ep } & -0.00152 & 0.030 \\
\hline & & $(0.00065)$ & \\
\hline \multirow{2}{*}{\multicolumn{2}{|c|}{ Constant }} & 0.226401 & \\
\hline & & $(0.04694)$ & \\
\hline \multicolumn{2}{|l|}{ Observations } & 32 & \\
\hline \multicolumn{2}{|l|}{ R-squared } & 0.8333 & \\
\hline \multicolumn{2}{|c|}{ Erroeres Estand en paréntesis } & & \\
\hline \multicolumn{2}{|l|}{ * Significante al $5 \%$} & & \\
\hline
\end{tabular}


Anexo 6. Estimación 4: Probit departamental 2005

\begin{tabular}{|c|c|c|}
\hline \multirow{2}{*}{$\begin{array}{l}\text { Efecto Departamental Variable } \\
\text { Educación }\end{array}$} & \multicolumn{2}{|c|}{ Coefficient (Std. Err.) } \\
\hline & epo & -0.01822 \\
\hline & & $(0.00823)$ \\
\hline & epno & 0.022047 \\
\hline & & $(0.01826)$ \\
\hline & аро & 0.000832 \\
\hline & & $(0.00040)$ \\
\hline & apno & -0.00296 \\
\hline & & $(0.00145)$ \\
\hline & eso & 0.046626 \\
\hline & & $(0.02891)$ \\
\hline & esno & 0.079833 \\
\hline & & $(0.04915)$ \\
\hline \multirow[t]{4}{*}{ Población } & pob & -0.00005 \\
\hline & & $(0.000030$ \\
\hline & imorm & 0.203466 \\
\hline & & $(0.29480)$ \\
\hline \multirow[t]{4}{*}{ Violencia } & homcom & -0.00310 \\
\hline & & $(0.00402)$ \\
\hline & terror & 0.072593 \\
\hline & & $(0.09483)$ \\
\hline \multirow{2}{*}{\multicolumn{2}{|c|}{ Constant }} & 0.026786 \\
\hline & & $(0.02457)$ \\
\hline Observations & & 32 \\
\hline \multicolumn{2}{|l|}{ Errores Estandar en paréntesis } & \\
\hline * Significancia al 5\% & & \\
\hline
\end{tabular}


Anexo 7. Estimación 5: Panel data 2003, 2004, 2005

\begin{tabular}{|c|c|c|c|}
\hline \multirow{2}{*}{ Efecto Departamental } & \multirow{2}{*}{ Variable } & \multicolumn{2}{|c|}{ Estimación } \\
\hline & & $1^{*}$ & $2^{*}$ \\
\hline \multirow[t]{16}{*}{ Educación } & epo & -0.000018 & -0.000031 \\
\hline & & $(0.00004)$ & $(0.00053)$ \\
\hline & epno & -0.000010 & 0.000011 \\
\hline & & $(0.00002)$ & $(0.00001)$ \\
\hline & apo & -0.000030 & 0.000030 \\
\hline & & (0.00052) & $(0.00041)$ \\
\hline & apno & -0.000014 & -0.000020 \\
\hline & & $(0.000018)$ & $(0.00001)$ \\
\hline & eso & 0.0000182 & -0.000040 \\
\hline & & $(0.00081)$ & $(0.00071)$ \\
\hline & esno & 0.000213 & 0.000331 \\
\hline & & $(0.00081)$ & $(0.00071)$ \\
\hline & aso & -0.000011 & -0.000016 \\
\hline & & $(0.00001)$ & $(0.00008)$ \\
\hline & asno & -0.000060 & 0.000018 \\
\hline & & $(0.00002)$ & $(0.00008)$ \\
\hline \multirow[t]{6}{*}{ Población } & rae & -0.000051 & 0.000047 \\
\hline & & $(0.00004)$ & $(0.00009)$ \\
\hline & papib & 18.84284 & 11.42992 \\
\hline & & (23.1199) & (11.03062) \\
\hline & pob & 0.000040 & 0.000010 \\
\hline & & $(0.000016)$ & $(0.000072)$ \\
\hline \multirow[t]{8}{*}{ Violencia } & homcom & 0.000290 & 0.000118 \\
\hline & & $(0.00041)$ & $(0.00021)$ \\
\hline & secuest & -0.003235 & 0.000304 \\
\hline & & $(0.00374)$ & $(0.00308)$ \\
\hline & extors & 0.003116 & 0.002371 \\
\hline & & (0.00298) & (0.00223) \\
\hline & terror & 0.019204 & 0.009827 \\
\hline & & $(0.00464)$ & $(0.00299)$ \\
\hline \multicolumn{4}{|c|}{$\begin{array}{l}\text { P-valor en paréntesis } \\
\text { * Fixed effects } \\
\text { Nivel de significancia de las estimaciones } 5 \% \\
\begin{array}{ll}\text { Test. de Haussman } & \text { Prob }>\mathrm{chi}^{2}=0.0043\end{array}\end{array}$} \\
\hline
\end{tabular}




\section{Apéndice: Normalización de variables}

A partir de un rango establecido para la variable, se define un intervalo continuo.

$$
\begin{aligned}
& X=\left[x_{\max }, x_{\text {min }}\right] \\
& X_{n}=[0,1]
\end{aligned}
$$

Con dicho intervalo, se obtiene la variable normalizada

$$
\begin{aligned}
& K x_{\text {min }}+P=-x_{\text {norm }} \\
& K x_{\text {max }}+P=-x_{\text {norm }}
\end{aligned}
$$

Siendo,

$$
\begin{aligned}
& K x_{\text {min }}+P=0 \\
& K x_{\text {max }}+P=1
\end{aligned}
$$

Donde,

$$
K=\frac{n x_{\text {norm }}}{x_{\text {max }-} x_{\min }}
$$

Siendo $n$ : número de departamentos

$$
P=-\left[\frac{x_{\max +} x_{\min }}{x_{\min -} x_{\max }}\right] x_{n o r m}
$$

Resultando la variable normalizada de la forma

$$
X_{\text {norm }}=\left(\sum_{i=1}^{n} x_{1} K\right)+P
$$

Con la cual, se puede establecer una variable que siga el comportamiento estocástico continuo de la variable original y al mismo tiempo permita realizar comparaciones con otras variables en intervalos continuos. 